\title{
Transient Global Amnesia: Risk Factors, Imaging Features, and Prognosis
}

\author{
Shunpo $\mathrm{He}^{\prime}$ \\ Zhenzhen $\mathrm{Ye}^{\prime}$ \\ Qingwei Yang ${ }^{2}$ \\ Jianzhong Lin $^{2}$ \\ Xingyu Chen ${ }^{2}$ \\ Zhongjie Chen ${ }^{2}$ \\ Liangyi Chen ${ }^{2}$ \\ 'The Graduate School of Fujian Medical \\ University, Fuzhou, People's Republic of \\ China; ${ }^{2}$ Department of Neurology, \\ Zhongshan Hospital, Xiamen University, \\ Xiamen, People's Republic of China
}

Correspondence: Qingwei Yang; Xingyu Chen

Department of Neurology, Zhongshan Hospital, Xiamen University, No. 203 of Hubin South Road, Siming District, Xiamen, 36I00I, People's Republic of China

Tel +86 592-2292113

Fax +86 592-2590120

Email yamgqimgwei@aliyun.com;

chemximgyu@163.com
Background and Aim: Transient global amnesia (TGA) was first described by Bender in 1956 and is characterized by sudden, temporary, and anterograde memory loss. This study aimed to explore the possible mechanisms of and lesions responsible for TGA.

Methods: Retrospective data were collected from all patients with TGA admitted to Zhongshan Hospital, affiliated with Xiamen University, between October 1, 2011, and October 30, 2018. Information about the TGA condition, previous history, and clinical examination of the TGA and control groups was recorded. Functional magnetic resonance imaging was performed on the patients to explore the possible lesions responsible for TGA.

Results: A total of 73 patients with TGA and 73 age- and gender-matched controls were included in the analysis. The differences in the migraine history $(9 / 2, p=0.038)$ were statistically significant in both groups, but no statistically significant difference was observed regarding the history of hypertension, diabetes, and other diseases. In addition, seven patients with TGA had lesions located in the hippocampal CA1 region; the dome column and hippocampal CA1 region exist in the same functional loop and play a synergistic role. The average follow-up period in the groups was 36 months. During the follow-up period, no significant differences in cerebral infarction, cerebral hemorrhage, CHD, or TGA attack between the groups were observed.

Conclusion: Migraine may be a risk factor of TGA, and cerebral infarction may be one of the pathogeneses. The brain area responsible for TGA may involve a memory loop comprising the hippocampal CA1 region and the fornix column among other parts.

Keywords: transient global amnesia, risk factors, magnetic resonance imaging, responsible lesions, prognosis

\section{Introduction}

Transient global amnesia (TGA) is a rare syndrome characterized by sudden onset of anterograde amnesia that universally lasts for up to $24 \mathrm{~h} .^{1}$ The exact pathogenesis of TGA is unknown. ${ }^{2}$ Nevertheless, various etiological hypotheses, including arterial ischemia, migraine, venous reflux disorder, and seizure, have been presented. The arterial ischemia theory is dominant, so TGA is classified as a transient ischemic attack. It is assumed that TGA is a clinical syndrome and that its responsibility is not limited to the hippocampus. In this research, a case-control study is conducted on patients with TGA, and functional magnetic resonance imaging (fMRI) is performed to explore the possible pathogeneses, responsible lesions, and long-term prognoses of TGA. 


\section{Patients and Methods}

\section{Patients}

This retrospective study involved data from patients with TGA admitted to Zhongshan Hospital, affiliated with Xiamen University, between October 1, 2011, and October 30, 2018. The control group, with matching age and sex, was enrolled in the physical examination department of the same hospital; the age was matched by \pm 1 year.

The diagnostic criteria were as follows: 1) anterograde amnesia; 2) witnessed by an observer; 3) no loss of consciousness or personal identity; 4) no other cognitive impairments besides amnesia; 5) no recent history of head trauma or seizure; and 6) symptoms that abate within $24 \mathrm{~h}^{3,4}$ The exclusion criteria were: 1) hypoglycemia at onset; 2) severe liver and kidney dysfunction; and 3) history of malignant tumor.

The inclusion criteria of the control group were: 1) underwent physical examination in the same period and 2) consented to follow-up. The exclusion criterion was meeting any one of the following: 1) moderate cognitive dysfunction during physical examination; 2) history of TGA; 3) severe liver and kidney dysfunction; and 4) history of malignant tumor.

\section{Data Collection}

The data collected in this study were as follows: 1) demographic data, including age, sex, and patient's dominant hand; 2) past medical history, including hypertension, diabetes, hyperlipidemia, atrial fibrillation, stroke, migraine, TGA attacks, and seizures; 3) clinical features related to TGA attacks, including inducing factors (dizziness, Valsalva movement, emotional agitation) and duration; 4) clinical and laboratory information, including admission blood glucose and lipids and auxiliary examinations, such as electrocardiogram, electroencephalogram (EEG), computed tomography (CT), jugular artery and vein ultrasound, MRI (TIWI, T2WI, DWI, fluid-attenuated inversion recovery [FLAIR], pattern deformation technique for hippocampal zoning, and probabilistic fiber tracer), and brain vascular evaluation (CT angiography [CTA], CT perfusion $[\mathrm{CTP}]$, and magnetic resonance angiography [MRA]); and 5) follow-up content, including survival, cerebral infarction, cerebral hemorrhage, TGA attack, and congestive heart disease. The termination points were as follows: patients died due to any reason or the follow-up time reached the end point (June 30, 2019).

\section{MRI Data Processing}

Three-dimensional (3D) structural imaging was performed in the patients with hippocampal lesions, and a graphic deformation technique was employed to divide the hippocampus into 3D structural images. Then, DWI was projected onto the structural images based on brain edge alignment to locate the specific lesion areas in the hippocampal region.

According to the marginal alignment of the brain, the subdivision of the hippocampus was projected onto diffusion tensor imaging [DTI], and a probabilistic fiber tracer was applied in the bilateral hippocampal CA1 region. This region was used as the seed point, and the bilateral fornix column was set as the target point to show the path of the nerve fibers in the region.

\section{Statistical Analyses}

Levene's test was applied to explore the type of data distribution for the descriptive data. A $t$-test was conducted to compare the variables between the TGA and control groups when the test statistics followed a normal distribution. Otherwise, a nonparametric test was conducted to compare the variables. Categorical variables were examined using a chi-square test or Fisher's exact test. All tests were two-sided. A value of $\mathrm{P}<0.05$ was considered statistically significant. All analyses were performed using SPSS 20.0.

\section{Results}

A total of 73 patients with TGA and the same number of matched controls were included in the study (Figure 1). The result of Levene's test was $p=0.818$ when the age between the TGA and control groups was compared, and the result was $p=0.901$ when the age between the patients with DWI lesions and controls was compared. A $t$-test was conducted to compare the age variable. The baseline data of the groups are presented in Table 1.

\section{Clinical Features of TGA}

The patients' symptoms were as follows: sudden onset of anterograde amnesia accompanied by repetitive questioning, lasting up to $24 \mathrm{~h}$. The following clinical features were related to TGA. Triggers occurred before the attack. In total, 21 patients herein had predisposing factors, including Valsalva movement before onset in 9 patients and emotional agitation in 12 patients. Regarding the duration of attack, the known duration of 49 patients 


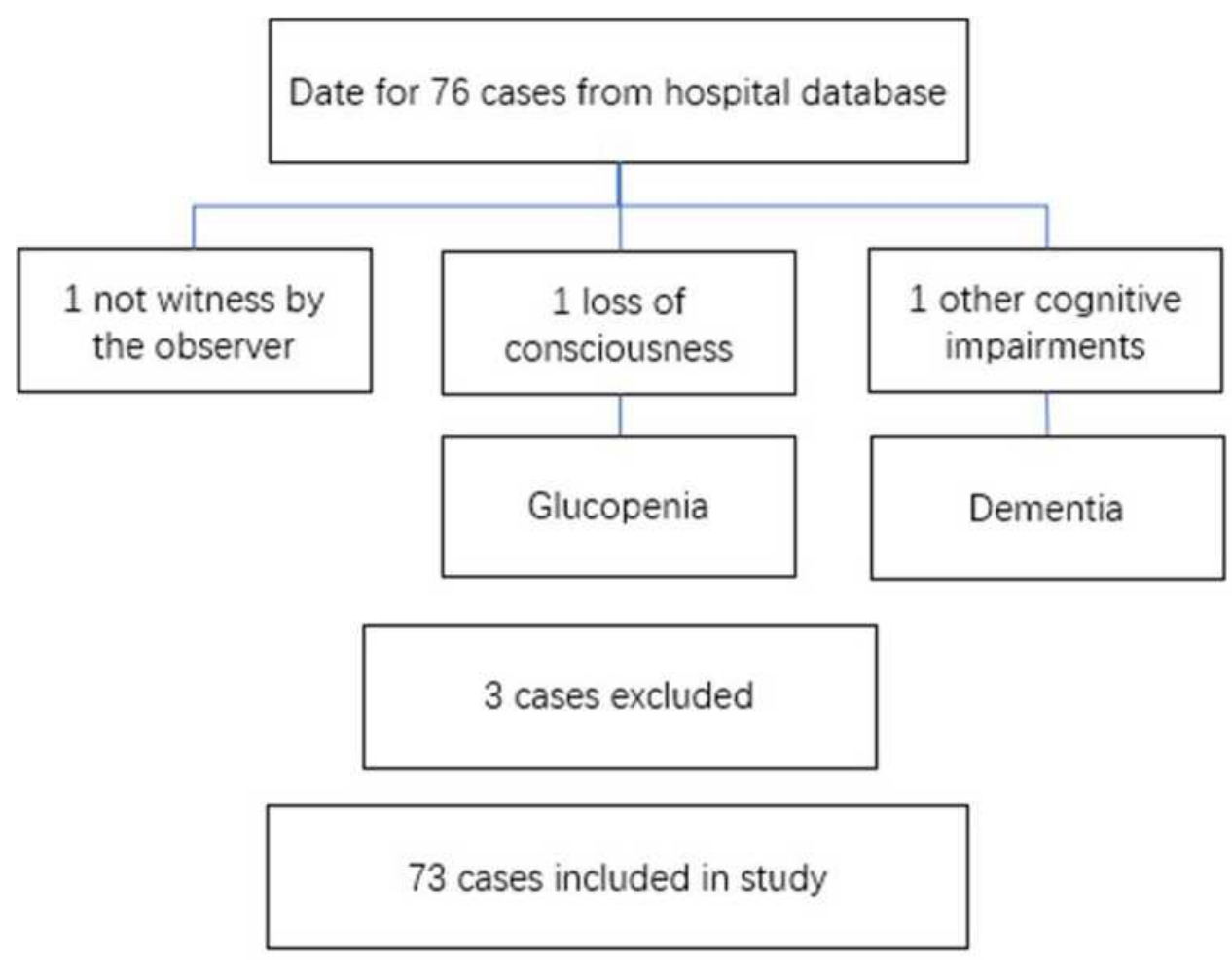

Figure I Patient selection flowchart.

ranged from $15 \mathrm{~min}$ to $13 \mathrm{~h}$, and the average duration was $4.5 \pm 2.8 \mathrm{~h}$.

\section{Examination Data}

In terms of the examination data, EEG was performed on only eight patients, and the results were normal. The symptoms of one of these lasted for three hours, and three days after the symptom recovery, this patient completed the EEG examination with normal results. In the fourth year of follow-up, the patient had a TGA relapse but was not admitted to a hospital for treatment and did not undergo EEG examination. In addition, one patient had symptoms lasting less than $30 \mathrm{~min}$; however, they did not undergo EEG examination, and there was no recurrence of TGA during follow-up.

Furthermore, 67 patients with TGA were examined via MRI within seven days after admission; 58 had normal DWI, and 9 had hyperintense lesions on DWI. In addition, seven patients had lesions in the hippocampus (two patients with bilateral hippocampal infarction), one patient had a lesion in the thalamus, and one patient had a lesion in the occipital lobe (Figure 2). The time to initial DWI from symptom onset was between 28 and $120 \mathrm{~h}$ in nine patients with a high signal on DWI.

\section{Hippocampal Subfields}

The hippocampus was segmented into 12 subregions; each section is shown in Figure 3. It was found that the DWI lesions were located in the hippocampal CA1 area.

\section{Nerve Fibers in the Hippocampal CAI Region}

The bilateral hippocampal CA1 region was used as the seed point, and the bilateral fornix column was set as the target point to show the path of the nerve fibers in the region (Figure 4). The results indicated that the nerve fibers in the bilateral hippocampal CA1 area converged to the bilateral fornix column.

\section{Vascular Assessment}

The vascular assessment results (CTA/CTP/MRA) of 67 patients with TGA were improved, and 59 patients were normal. However, eight patients had abnormal cerebral arteries: Three had severe stenosis of the M1 segment of 
Table I Clinical Information of 73 Patients with TGA and Control Group

\begin{tabular}{|l|l|l|l|}
\hline Characteristics & TGA & Controls & P \\
\hline Number & 73 & 73 & - \\
\hline Sex: Female & 49 & 49 & 1.000 \\
\hline Age(y), mean \pm SD & $62.0 \pm 8.5$ & $62.0 \pm 8.0$ & 0.545 \\
\hline Hypertension & 21 & 13 & 0.115 \\
\hline Diabetes & 11 & 12 & 1.000 \\
\hline Hyperlipidemia & 18 & 17 & 1.000 \\
\hline Atrial fibrillation & 3 & 1 & 0.625 \\
\hline Previous stroke & 3 & 1 & 0.625 \\
\hline History of migraine & 9 & 2 & 0.038 \\
\hline History of TGA & 3 & 0 & - \\
\hline Seizures/epilepsy & 0 & 0 & - \\
\hline Follow-up & 68 & 67 & 1.000 \\
\hline TGA & 1 & 0 & - \\
\hline Death & 1 & 0 & - \\
\hline Cerebral infarction & 1 & 1 & - \\
\hline Cerebral hemorrhage & 1 & 0 & 1.000 \\
\hline Congestive heart disease & 0 & 1 & - \\
\hline
\end{tabular}

the middle cerebral artery (the right side in two and the left side in one); three had steal syndrome; one had stage 3 Moyamoya disease; and one had arteriovenous malformation in the right occipital lobe. In addition, 2 of the 59 normal patients were subjected to CTP. One was still in the attack stage of TGA, and their symptoms had not yet ceased. The results showed that the medial side of the right temporal lobe and the basal ganglia had hypoperfusion, but no obvious vascular abnormality was found in this patient (Figure 5).

\section{Follow-Up}

Among the 73 patients with TGA, 68 completed the follow-up. The average follow-up period was $36.0 \pm 22.6$ months. Four patients had end events: one case of death, one case of recurrent TGA, one case of cerebral infarction, and one case of cerebral hemorrhage. In the control group, 67 patients completed the follow-up, among whom 1 had cerebral infarction and 1 had myocardial infarction.

\section{Analysis of Risk Factors}

Among the 73 patients with TGA, 9 patients with lesions on DWI were compared with the other patients in terms of risk factors for cerebrovascular disease (Table 2). The results showed that the differences between the groups were not statistically significant.

\section{Discussion}

\section{Hippocampal Ischemia/Infarction}

In the present study, DWI revealed that nine patients with TGA had hyperintense lesions (Figure 2), suggestive of cytotoxic edema in the hippocampus. Moreover, six of these patients were considered to have cerebral vascular disease (including patients with previous atrial fibrillation, low CTP perfusion, microembolism, and/or previous risk factors for vascular disease). Three of them were unable to be classified. These findings suggested that vascular disease is one of the pathogeneses of TGA. In addition, two patients with TGA were examined through CTP, which showed low perfusion in the medial temporal lobe (Figure 5). One patient's symptoms had not yet alleviated, but the patient had no obvious vascular abnormalities, indicating that vascular disease is a pathogenesis of TGA.

Previous studies have proposed temporal lobe ischemia $;^{5-7}$ however, many other studies do not support this mechanism, and several major reasons have been presented. First, patients with TGA have no increased risk factors of cerebrovascular disease compared with healthy controls. ${ }^{8-10}$ Consistent with previous studies, our study

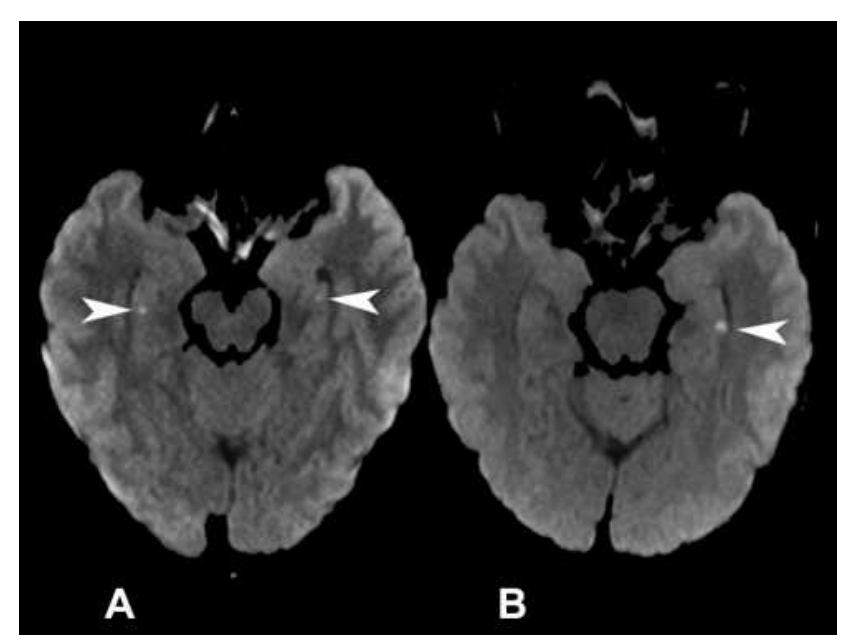

Figure 2 (A) DWI image of patient. The arrows point to DWI lesions in the hippocampal CAI area; (B) DWI image of patient. The arrows point to DWI lesions in the hippocampal CAI area. 


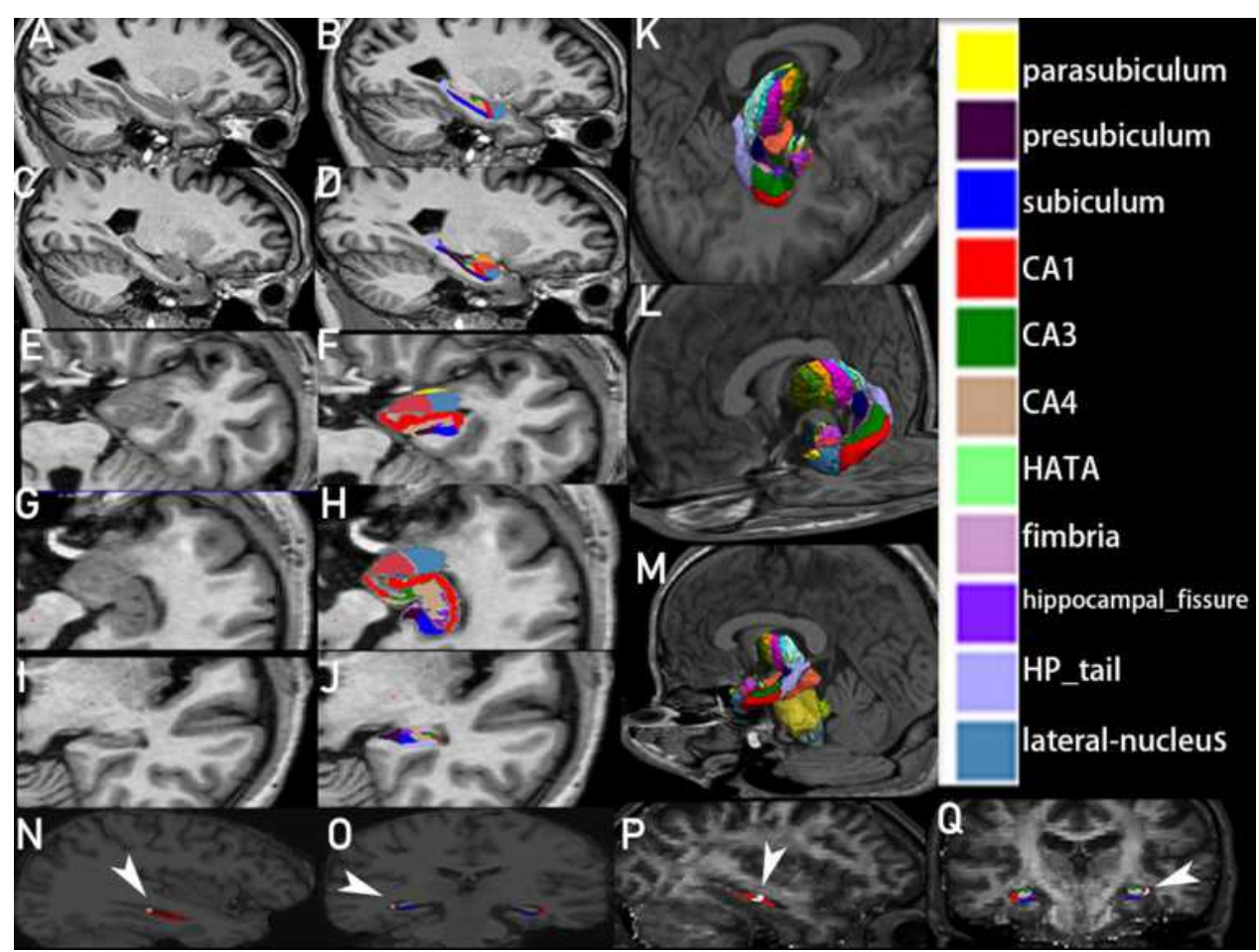

Figure 3 Hippocampal subdivision image. The highlights in the figure are high-signal lesions on DWI, showing bilateral hippocampal lesions located in the hippocampal CAI, as shown by the arrows. (A-J) showed the hippocampus from different planes. (K-M) showed a 3D partition map of the hippocampus from three directions. The (N-Q) white arrows indicate the CAI lesions in the hippocampus. The red area in (A-Q) is the hippocampal CAI area.

examined the risk of cerebrovascular events in both groups, and the results demonstrated that the patients with TGA did not have high risk factors of cerebrovascular disease. Second, the risk of cerebrovascular events in patients with TGA does not increase during the follow-up period, ${ }^{10-13}$ which is also supported by our research. During our follow-up period, no

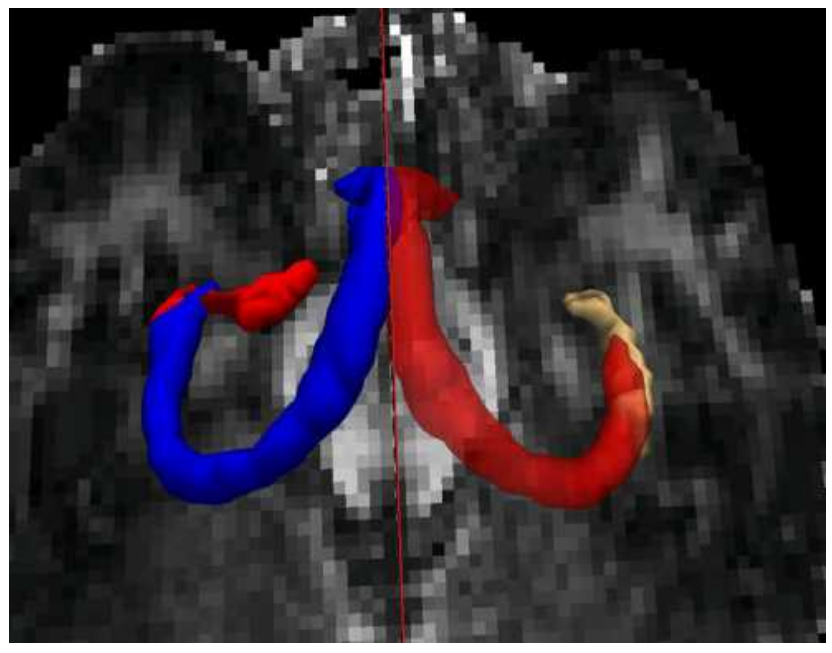

Figure $4 \mathrm{DTI}$ images of the hippocampal CAI region. Tracer of nerve fibers in the two-sided hippocampal CAI area, showing the bilateral fornix. statistically significant differences in cerebrovascular events between the TGA and control groups were observed. Third, although high signal lesions were detected in the hippocampus during DWI, the patients seemed to recover within two weeks. Finally, the patients' symptoms were alleviated rapidly, and the recurrence rate was low.

Considering these problems, we present the following findings: On the one hand, we found a case with a large number of micro-embolus signals detected during Valsalva motion through a transcranial Doppler (TCD) test. Moreover, this patient's heart ultrasound revealed a patent foramen ovale, and left hippocampal infarction was shown as a lesion on DWI. The CTA was normal in the cerebral vascular territories. These results suggested that a mechanism of TGA might be related to microembolization. Patients with cerebral infarction caused by embolism often have no risk factors of cerebrovascular disease. Therefore, this instance is not unique but has a counterpart. Cases of suspected microembolization in patients with TGA have been reported. ${ }^{14,15}$ On the other hand, our examination of the high signal intensity in the hippocampus on DWI revealed that the volume of a high signal focused on DWI was exited volatility, accounting 


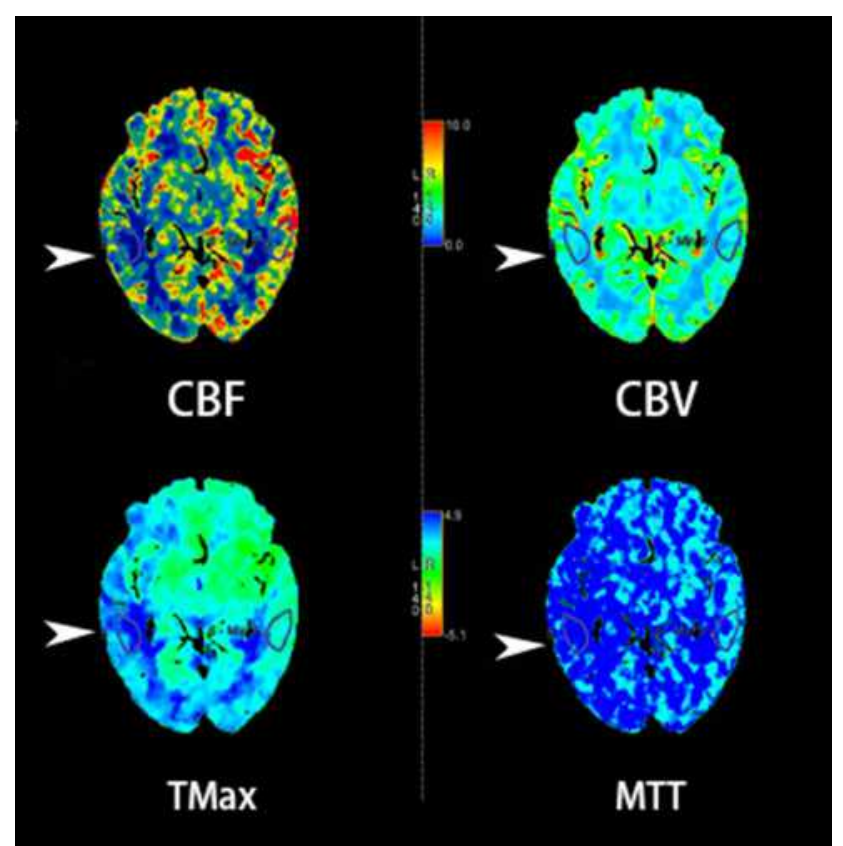

Figure 5 CTP images of patients at the onset stage. The figure shows that the right cerebral blood volume was normal, the cerebral blood flow decreased, and the peak time and mean transit time were extended. Therefore, local blood perfusion was possibly reduced.

Abbreviations: CBF, cerebral blood flow; CBV, cerebral blood volume; TMax, peak time; MTT, mean transit time.

for a low proportion of the total volume of the hippocampal CA1 region, eg, the volume of the lesions in the hippocampus in this study. ${ }^{16}$ Furthermore, the size of the

Table 2 In Patients of TGA, 9 Patients with DWI Lesions Were Compared to the Rest of the Patients

\begin{tabular}{|l|l|l|l|}
\hline Characteristics & DWI Lesions & No DWI Lesions & P \\
\hline Number & 9 & 58 & - \\
\hline Sex: Female & 6 & 40 & 1.000 \\
\hline Age(y), mean \pm SD & $59.7 \pm 8.3$ & $61.7 \pm 8.5$ & 0.534 \\
\hline Hypertension & 2 & 16 & 1.000 \\
\hline Diabetes & 1 & 8 & 1.000 \\
\hline Hyperlipidemia & 2 & 15 & 1.000 \\
\hline Atrial fibrillation & 1 & 2 & 0.356 \\
\hline Previous stroke & 0 & 2 & 1.000 \\
\hline History of migraine & 1 & 7 & 1.000 \\
\hline History of TGA & 1 & 2 & 0.356 \\
\hline Seizure/epilepsy & 0 & 0 & - \\
\hline
\end{tabular}

Abbreviations: TGA, transient global amnesia; SD, standard deviation; DWI, MR diffusion-weighted imaging. lesions ranged from 2.22 to $64.9 \mathrm{~mm}^{3}$ (mean $=22.3 \mathrm{~m}^{3}$ ). In another study, 11 patients showed punctate foci of restricted diffusion in the hippocampus (mean size $=$ $3.7 \mathrm{~mm}$; range $=2.0-6.5 \mathrm{~mm}) .{ }^{17}$ This observation was confirmed in our research. In particular, the lesions in the hippocampus of seven patients were only punctate. Moreover, DWI revealed lesions in the hippocampus, and FLAIR indicated lesions in several patients but no lesions in others. Additionally, DWI showed cytotoxic edema. When cell injury leads to abnormal metabolism, a high signal may be shown on DWI. When the damage affects the tissue, FLAIR exhibits a high signal, indicating that the hippocampus was damaged during the TGA attack. When some of the damage factors disappear quickly, the damage does not involve the tissue, and the FLAIR sequence will not show high signal intensity. After the onset of TGA, the hippocampus is damaged, as supported by previous studies, ${ }^{16,18,19}$ which also proposed that the damage elicits an effect after the onset.

In summary, our study indicated that several of the patients with TGA had hippocampal ischemic injury and that the possible mechanisms involved microembolization and arterial spasm among other processes. The overall function of the hippocampus could be recovered quickly since the proportion of injury was small. Previous studies have found that the hippocampus has metabolic vulnerability. ${ }^{18}$ In metabolic vulnerability, the lower the threshold, the easier it is for damage to occur. With the occurrence of necrosis or tolerance in hippocampal cells with a low threshold, the recurrence decreases. Although these assumptions are reasonable, further evidence is needed to prove them.

\section{Lesions on DWI}

The patients with TGA and lesions on DWI were further examined using fMRI. Similar to the results of previous studies, ${ }^{12,16,17,20-25}$ our findings showed that the lesions on DWI were mainly located in the hippocampal CA1 area (Figure 3). Seven of the patients with TGA and hippocampal lesions on DWI were right-handed, suggesting that the left cerebral hemisphere was dominant. The DWI sequence showed that the fewest lesions were in the left hippocampal CA1 region (including two bilateral hippocampal lesions and five simple left hippocampal CA1 lesions). This suggested that transient memory processing in the hippocampal CA1 region might exist predominantly in the hippocampus or be functionally differentiated in the bilateral hippocampus. Several studies have also proposed that the function of the bilateral 
hippocampus differs and that damage in the left hippocampal CA1 region leads to memory impairment. ${ }^{26}$

\section{Functions of Hippocampal CAI Area}

Previous studies have shown that the hippocampal CA1 area has several main functions, ${ }^{20,27,28}$ including immediate memory processing and memory retrieval and reproduction. This information is also consistent with clinical findings. Although some patients have immediate memory disorders, other disorders may occur in the retrieval and reproduction of long-term memory. Nevertheless, not all long-term memory retrieval and reproduction are involved. Other loops may participate in long-term memory extraction and reproduction, and the hippocampal CA1 area is mainly implicated in processing immediate memory.

\section{The Brain Area Responsible for TGA}

Probabilistic fiber tracking was performed in the patients with lesions in the bilateral hippocampal CA1 area; the results showed that the nerve fibers of the bilateral hippocampal CA1 convened to the fornix column. This phenomenon explained a previously reported case, ${ }^{19}$ which indicated that fornix column infarction could lead to TGA attack. Our study structurally supported that the hippocampal CA1 region and the fornix column are in the same functional loop and provided a basis for stating that amnesia is caused by fornix column infarction. This type of amnesia has characteristics of TGA and other conditions since it may also involve the function of the fornix column in other functional loops. The fornix column may have a synergistic effect with the hippocampal CA1 region. In addition, recent research has demonstrated the association of the hippocampal CA1 area with the ventral tegmental area and longterm memory during associative encoding tasks. ${ }^{27}$ In contrast, the functional connectivity between the hippocampal CA1 area and DG/CA3 is greater during retrieval tasks than during encoding tasks; the strength of this connectivity is significantly correlated with retrieval success. Other studies have reported that the hippocampal CA1 area transfers the activity of the CA3 area to other brain regions within the hippocampal trisynaptic pathway as the main relay station. ${ }^{29}$ Therefore, our results suggested that the hippocampal CA1 region might act as a relay station in memory processing and transfer information from the CA3 area or other areas to other brain regions through the fornix column. The focus in this pathway could destroy the integrity of the loop and lead to a TGA attack.

\section{Migraine}

Our study found a significant difference in the history of migraine between the groups (Table 1). Similar to the findings of previous studies, ${ }^{30}$ our results supported that migraine is a risk factor of TGA. We also found that the patients with migraine were more likely to develop TGA $(\mathrm{IRR}=2.48, \mathrm{p}=0.002)$. It has been recognized that cortical spreading depression exists in the pathological mechanism of migraine. Several studies ${ }^{30}$ have suggested that this could explain why patients with migraine are likely to suffer from TGA: Cortical spreading depression inhibits neuronal activity by changing the excitability of neurons in the hippocampal CA1 region, possibly leading to CA1 neuronal damage. ${ }^{31,32}$

\section{Valsalva Movement}

Some patients with TGA have Valsalva movement before onset, which might be an inducing factor of TGA. Several studies have proposed that Valsalva movement may lead to venous blood reflux disturbance. ${ }^{33,34}$ Therefore, it might be one of the inducing factors of TGA. It is attributed to cerebral venous reflux disorder in the temporal lobe and also causes an increase in pressure in the chest cavity, thereby hindering the reflux of the internal jugular vein to the superior vena cava. Retrograde blood flow occurs in the jugular vein when patients have jugular vein insufficiency; venous hypertension in the medial temporal lobe is subsequently observed, possibly causing TGA. ${ }^{33,34}$ Other studies have reported that patients with TGA have a high rate of abnormal internal jugular vein. ${ }^{35,36}$ Unfortunately, most patients in the current study did not undergo jugular ultrasonography, and we failed to reveal possible jugular vein insufficiency in the patients with Valsalva movement before the disease onset. However, as mentioned earlier, in one patient, a large number of micro-embolus signals were detected in retrograde during Valsalva movement in the TCD test. This patient's heart ultrasound also revealed a patent foramen ovale.

In summary, Valsalva movement might induce TGA. The possible pathogenesis may involve venous hypertension in the medial temporal lobe or abnormal embolism. Therefore, patients with TGA, especially those with Valsalva movement before onset, should have improved ultrasound of their jugular vein and heart as well as a TCD 
test to detect jugular vein insufficiency and abnormal embolism.

\section{Epilepsy}

Although several studies have reported an abnormal EEG in patients with TGA, they failed to diagnose epilepsy. ${ }^{21,37}$ In most studies, the EEG of patients with TGA returns to normal after the patients have recovered from TGA, so the findings are not sufficiently convincing. In our study, eight patients underwent EEG, and their results were normal. Importantly, for one patient, this was during a TGA attack, and the symptom was not alleviated. Furthermore, this patient's EEG results were normal, so our study does not support epilepsy as a pathogenesis. However, TGA should be distinguished from transient epileptic amnesia (TEA). The time of onset of TEA is short (usually a few minutes), and it is often a recurrent attack. It may be accompanied by oral automatism and olfactory or gustatory hallucinations. Moreover, TEA is characterized by an abnormal EEG in the temporal or frontotemporal regions. Therefore, patients with TGA should have a refined EEG to exclude TEA if their symptoms last for a short time and occur repeatedly.

\section{Limitations}

In retrospective data collection, information may be lost, as we experienced in our research. Several control aspects were carried out: After the data were collected, they were proofread by another researcher. Then, during follow-up, inaccurate information was confirmed with the patients and their families. Uncertain information was excluded from the statistical analysis, and vulnerable indicators were avoided in the discussion. Therefore, these differences did not affect our main results.

A memory scale was not used to evaluate the memory of the patients with TGA in our study. In a previous study, microstructural sequelae were still detected in the hippocampus after TGA. ${ }^{16}$ In addition, recent research has shown that patients' memory behavior still seems abnormal after recovery from the symptoms of TGA. ${ }^{20}$ Therefore, in the future, a memory scale should be used to quantitatively evaluate whether the sequelae of memory exist in patients with TGA and further elucidate this condition.

\section{Conclusion}

Migraine may be a risk factor of TGA, and cerebral infarction may be one of the pathogeneses. The brain area responsible for TGA may involve a memory loop comprising the hippocampal CA1 region, the fornix column, and other parts. After a TGA attack, the long-term prognosis is good.

\section{Ethics Approval and Consent to Participate}

We confirm that we have read the Editorial Policy pages. This study was conducted with approval from the Ethics Committee of Zhongshan hospital, Xiamen University. Patient consent to review their medical records was required by the Ethics Committee of Zhongshan hospital, Xiamen University. And written informed consent was obtained from all participants. This study was conducted in accordance with the declaration of Helsinki.

\section{Disclosure}

The authors report no conflicts of interest in this work.

\section{References}

1. Bender MB. Single episode of confusion with amnesia. Bull N Y Acad Med. 1960;36:197-207.

2. Arena JE, Rabinstein AA. Transient global amnesia. Mayo Clin Proc. 2015;90(2):264-272. doi:10.1016/j.mayocp.2014.12.001

3. Caplan L, Chedru F, Lhermitte F, et al. Transient global amnesia and migraine. Neurology. 1981;31(9):1167-1170. doi:10.1212/ WNL.31.9.1167

4. Hodges JR, Warlow CP. Syndromes of transient amnesia: towards a classification. A study of 153 cases. J Neurol Neurosurg Psychiatry. 1990;53(10):834-843. doi:10.1136/jnnp.53.10.834

5. Michel P, Beaud V, Eskandari A, et al. Ischemic amnesia: causes and outcome. Stroke. 2017;48(8):2270-2273. doi:10.1161/ STROKEAHA.117.017420

6. Himeno T, Kuriyama M, Takemaru M, et al. Vascular risk factors and internal jugular venous flow in transient global amnesia: a Study of 165 Japanese Patients. J Stroke Cerebrovasc Dis. 2017;26 (10):2272-2278. doi:10.1016/j.jstrokecerebrovasdis.2017.05.010

7. Jang J-W, Park SY, Hong J-H, et al. Different risk factor profiles between transient global amnesia and transient ischemic attack: a large case-control study. Eur Neurol. 2014;71(1-2):19-24. doi:10.1159/000354023

8. Easton JD, Saver JL, Albers GW, et al. Definition and evaluation of transient ischemic attack: a scientific statement for healthcare professionals from the American Heart Association/American Stroke Association Stroke Council; Council on Cardiovascular Surgery and Anesthesia; Council on Cardiovascular Radiology and Intervention; Council on Cardiovascular Nursing; and the Interdisciplinary Council on Peripheral Vascular Disease. The American Academy of Neurology affirms the value of this statement as an educational tool for neurologistsss. Stroke. 2009;40(6):2276-2293. doi:10.1161/ STROKEAHA.108.192218

9. Felix MM, Castro LH, Maia AC Jr, da Rocha AJ. Evidence of acute ischemic tissue change in transient global amnesia in magnetic resonance imaging: case report and literature review. $J$ Neuroimaging. 2005;15(2):203-205. doi:10.1111/j.1552-6569.2005.tb00309.x

10. Matsui M, Imamura T, Sakamoto S, et al. Transient global amnesia: increased signal intensity in the right hippocampus on diffusion-weighted magnetic resonance imaging. Neuroradiology. 2002;44(3):235-238. doi:10.1007/s002340100699 
11. Arena JE, Brown RD, Mandrekar J, et al. Long-term outcome in patients with transient global amnesia: a Population-sBased Study. Mayo Clin Proc. 2017;92(3):399-405. doi:10.1016/j. mayocp.2016.11.015

12. Mangla A, Navi BB, Layton K, et al. Transient global amnesia and the risk of ischemic stroke. Stroke. 2014;45(2):389-393. doi:10.1161/ STROKEAHA.113.003916

13. Pantoni L, Bertini E, Lamassa M, et al. Clinical features, risk factors, and prognosis in transient global amnesia: a follow-up study. Eur J Neurol. 2005;12(5):350-356. doi:10.1111/j.1468-1331.2004.00982.x

14. Stollberger C, DeCillia N, Finsterer J. Tako-tsubo cardiomyopathy with transient global amnesia and cerebellar embolic stroke triggered by existential fear. Neurol Neurochir Pol. 2018;52(3):394-396. doi:10.1016/j.pjnns.2017.12.013

15. Santos S, López Del Val J, Tejero C, et al. Transient global amnesia: a review of 58 cases. Rev Neurol. 2000;30(12):1113-1117.

16. Wang X, Zhang R, Wei W, et al. Long-term sequelae of hippocampal lesions in patients with transient global amnesia: a multiparametric MRI study. J Magn Reson Imaging. 2018;47(5):1350-1358. doi:10.1002/jmri.25844

17. Jain TP, Patel R, Gawarikar Y. Transient global amnesia: diffusion MRI findings. Indian J Radiol Imaging. 2018;28(1):6-9. doi:10.4103/ ijri.IJRI_12_17

18. Bartsch T, Döhring J, Reuter S, et al. Selective neuronal vulnerability of human hippocampal CA1 neurons: lesion evolution, temporal course, and pattern of hippocampal damage in diffusion-weighted MR imaging. J Cereb Blood Flow Metab. 2015;35(11):1836-1845. doi:10.1038/jcbfm.2015.137

19. Paech D, Kuder TA, Roßmanith C, et al. What remains after transient global amnesia (TGA)? An ultra-high field $7 \mathrm{~T}$ magnetic resonance imaging study of the hippocampus. Eur J Neurol. 2019. doi:10.1111/ ene. 14099

20. Hanert A, Pedersen A, Bartsch T. Transient hippocampal CA1 lesions in humans impair pattern separation performance. Hippocampus. 2019;29(8):736-747. doi:10.1002/hipo.23073

21. Imperatori C, Farina B, Todini F, et al. Abnormal EEG power spectra in acute transient global amnesia: a quantitative EEG Study. Clin EEG Neurosci. 2018:1550059418780780. doi:10.1177/15500594 18780780

22. Jeong M, Jin J, Kim JH, et al. Incidental hippocampal hyperintensity on diffusion-weighted MRI: individual susceptibility to transient global amnesia. The Neurologist. 2017;22(4):103-106. doi:10.1097/ NRL.0000000000000123

23. Kim J, Kwon Y, Yang Y, et al. Clinical experience of modified diffusion-weighted imaging protocol for lesion detection in transient global amnesia: an 8-year large-scale clinical study. J Neuroimaging. 2014;24(4):331-337. doi:10.1111/jon.12021

24. Moon Y, Moon WJ, Han SH. The structural connectivity of the recurrent transient global amnesia. Acta Neurol Scand. 2016;134 (2):160-164. doi:10.1111/ane.12518
25. Webb AJ, Rothwell PM. Neurological picture. Transient global amnesia associated with bilateral restricted diffusion in the lateral hippocampus. J Neurol Neurosurg Psychiatry. 2013;84(5):522. doi:10.1136/jnnp-2012-304542

26. Gupta M, Kantor MA, Tung CE, et al. Transient global amnesia associated with a unilateral infarction of the fornix: case report and review of the literature. Front Neurol. 2014;5:291. doi:10.3389/ fneur.2014.00291

27. Duncan K, Tompary A, Davachi L. Associative encoding and retrieval are predicted by functional connectivity in distinct hippocampal area CA1 pathways. $J$ Neurosci. 2014;34(34):11188-11198. doi:10.1523/JNEUROSCI.0521-14.2014

28. Kesner RP, Morris AM, Weeden CSS. Spatial, Temporal, and Associative Behavioral Functions Associated with Different Subregions of the Hippocampus. 2012; Oxford, UK: Oxford Handbooks Online /The Oxford Handbook of Comparative Cognition (2 ed.).

29. Knierim JJ, Neunuebel JP. Tracking the flow of hippocampal computation: pattern separation, pattern completion, and attractor dynamics. Neurobiol Learn Mem. 2016;129:38-49. doi:10.1016/j. nlm.2015.10.008

30. Lin K-H, Chen Y-T, Fuh J-L, et al. Migraine is associated with a higher risk of transient global amnesia: a nationwide cohort study. Eur J Neurol. 2014;21(5):718-724. doi:10.1111/ene.12346

31. Schwedt TJ, Dodick DW. Advanced neuroimaging of migraine. Lancet Neurol. 2009;8(6):560-568. doi:10.1016/S1474-4422(09) 70107-3

32. Bartsch T, Deuschl G. Transient global amnesia: functional anatomy and clinical implications. Lancet Neurol. 2010;9(2):205-214. doi:10.1016/S1474-4422(09)70344-8

33. Han K, Zhao Y, Zhang F, et al. The correlation between venous reflux disorder andtransient global amnesia. Chin J Stroke. 2016;11 (07):547-555.

34. Han $\mathrm{K}, \mathrm{Hu}-\mathrm{H}-\mathrm{H}$, Chao A-C, et al. Transient global amnesia linked to impairment of brain venous drainage: an ultrasound investigation. Front Neurol. 2019;10:67. doi:10.3389/fneur.2019.00067

35. Agosti C, Borroni B, Akkawi NM, et al. Cerebrovascular risk factors and triggers in transient global amnesia patients with and without jugular valve incompetence: results from a sample of 243 patients. Eur Neurol. 2010;63(5):291-294. doi:10.1159/000292502

36. Cejas C, Cisneros LF, Lagos $\mathrm{R}$, et al. Internal jugular vein valve incompetence is highly prevalent in transient global amnesia. Stroke. 2010;41(1):67-71. doi:10.1161/STROKEAHA.109.566315

37. Ren X, Yang F, Liu C, et al. Electroencephalogram analysis of 20 cases of transientglobal amnesia. J Clin Exp Med. 2012;11(09):698699.
Neuropsychiatric Disease and Treatment

\section{Publish your work in this journal}

Neuropsychiatric Disease and Treatment is an international, peerreviewed journal of clinical therapeutics and pharmacology focusing on concise rapid reporting of clinical or pre-clinical studies on a range of neuropsychiatric and neurological disorders. This journal is indexed on PubMed Central, the 'PsycINFO' database and CAS, and is the official journal of The International Neuropsychiatric Association (INA). The manuscript management system is completely online and includes a very quick and fair peer-review system, which is all easy to use. Visit http://www.dovepress.com/testimonials.php to read real quotes from published authors. 